\section{Coordinating vaccine use is best way to combat polio}

SIR - In their Commentary article "A global call for new polio vaccines" (Nature 434, 699; 2005), David L. Heymann, Roland W. Sutter and R. Bruce Aylward argued for vaccines to be developed and stockpiled. I propose, instead, that there is no need for new polio vaccines, but rather that more sensible and coordinated use is needed of the two major vaccines in existence.

One of these is the inactivated Salk vaccine and the other the live, but attenuated, Sabin vaccine (also known as oral polio vaccine). The Salk vaccine is safe, and was used to eradicate polio in Sweden. The Sabin vaccine is not so safe: for many years in the United States most of the polio cases that did occur were shown to have originated from the Sabin vaccine, owing to reversion of the attenuated viruses.

At the time both vaccines were introduced (a little over 50 years ago), I was an employee of the Lilly Research Laboratories of Eli Lilly and was intimately involved in production of the material for Salk's clinical trial.

Even in those days, I could not understand why a decision was never made, from a public health viewpoint, to insist that all populations slated for vaccination should receive one or possibly two shots of the inactivated Salk vaccine before receiving the live Sabin vaccine. The live vaccine has the advantage of multiplying in the gut, the normal site of polio multiplication, and thus triggering a greater reaction by the host's immune system. This normal intestinal route of infection was one reason swimming pools were considered to be such a hazard. However, if the host had first been protected by the Salk vaccine the risk posed by the Sabin dose would be reduced.

I still believe that this is more sensible from a public health viewpoint and, today, one nolonger has to be concerned about the animosity between the vaccine developers or manufacturers.

Irving S. Johnson

4601 Rue Belle Mer, Sanibel, Florida 33957, USA

\section{Don't drop current vaccine until we have new ones}

SIR - David L. Heymann and colleagues, from the World Health Organization (WHO) issued a global call for new poliovirus vaccines in their recent Commentary article (Nature 434,$699 ; 2005)$. This represents a welcome change in the WHO poliovirus eradication strategy; however, some important issues remain to be addressed.

The global struggle against poliomyelitis has been a huge success and, ultimately, is expected to lead to eradication of the disease. However, several risk factors associated with the principal vaccine used today (oral polio vaccine, $O P V$ ), suggest that new vaccines will still be needed to accomplish eradication.

As Heymann and colleagues note, circulating vaccine-derived polioviruses have caused poliomyelitis outbreaks in five countries. Moreover, some people continue to excrete virulent poliovirus more than ten years after being vaccinated with OPV. Ending OPV immunization, therefore, can lead to increased risks from OPV derivatives, and Heymann and his colleagues are justified in calling for the development of new vaccines.

At present there are no adequate alternatives to trivalent OPV, but the WHO proposes to replace it with monovalent OPV (mOPV). Although mOPV vaccination may be useful under some circumstances, it poses the same risk factors as OPV. These risks vastly increase if mOPV is used in the postvaccination era, because of diminishing population immunity.

\section{"One no longer has to be concerned} about animosity between vaccine manufacturers." - Irving S. Johnson

Switching from OPV to the more costly Salk inactivated vaccine (IPV) eliminates these risks, and this has occurred in most industrialized countries. However, accidental release of the wild-type virus during IPV manufacture threatens to restart epidemics. Therefore, the WHO suggests using 'Sabin IPV' (sIPV) produced by inactivation of OPV. However, questions about the effectiveness of sIPV require further research and extensive clinical trials.

We propose instead that time should be given to conducting research for truly new poliovirus vaccines, and for the development of anti-polio drugs.

We also urge the WHO and public-health authorities around the world not to stop OPV vaccination until an efficacious and low-cost IPV has been developed.

We must also consider whether we should ever terminate poliovirus vaccination. In a world threatened with terrorism, we should remember that polioviruses could be synthesized rapidly at very low cost. The polio outbreak that happened in the 1940s in an Eskimo village in arctic Canada, with 25\% poliomyelitis and high mortality, provides a sobering example of the devastation that can occur in unvaccinated communities. Vadim L. Agol*, Konstantin Chumakovt, Ellie Ehrenfelds, Eckard Wimmer\$

*M. P. Chumakov Institute of Poliomyelitis and Viral Encephalitides, Russian Academy of Medical Sciences, Moscow Region 142782, Russia †Center for Biologics Evaluation and Research, Food and Drug Administration, 1401 Rockville Pike, Rockville, Maryland 20852, USA
† National Institute of Allergy and Infectious Diseases, NIH, Bethesda, Maryland 20892, USA $\S$ Department of Molecular Genetics and Micrabiology, Stony Brook University School of Medicine, Stany Brook, New York, USA

\section{Images: keep a distinction between beauty and truth}

SIR - Your News Feature "CSI: cell biology" (Nature 434, 952-953; 2005) addressed an insidious, but largely ignored, problem with undocumented image enhancement in scientific papers.

The enthusiasm for presenting the 'best' scientific image possible seems to be driven by a desire both to tell a clear story and to compose an aesthetically pleasing image. It would be helpful if all journals adopted a code of image-manipulation ethics, such as those described in the News Feature for The Journal of Cell Biology, to guide authors and reviewers alike.

After teaching extramural microscopy and imaging courses for a number of years, I have observed two additional factors that contribute to the widespread manipulation of scientific images.

First, graduate school curricula typically do not offer systematic instruction in microscopy or image formation, with the result that most biology graduate students rely on ad-hoc training by more senior students or postdocs. Without comprehensive training, many junior scientists are unable to produce the quality of image desired and resort to image software manipulation to ' $\mathrm{fix}$ ' the image. Developing expertise in image acquisition would be preferable to resorting to post-acquisition manipulation.

Second, your News Feature attributed the increase in questionable image manipulation practices to the eagerness of students and postdocs to improve their data.

However, in my courses, many trainees report that they are instructed - often pressured - by the principal investigator to produce images consistent with expectations. This often means losing the dynamic signal range inherent in biological material to create a high-contrast 'unambiguous' image. Such instructed manipulation, either in image acquisition or post-processing, essentially discards data at best and may be misleading at worst. Thus it is incumbent, not only on the scientist in training, but also on the scientist performing the training, to maintain high ethical standards while pursuing both beauty and truth.

Daniel A. Peterson

Department of Neuroscience,

Rosalind Franklin University of Medicine

and Science, 3333 Green Bay Road,

North Chicago, Illinois 60064, USA 